\title{
GUIDELINES FOR CONTRIBUTORS
}

Technostyle publishes articles, notes and commentaries for instructors and practitioners in the fields of technical communication and business communication. Articles present research findings and in-depth analyses, while notes are narrower in scope and focus on a specific teaching or writing technique. All submissions to Technostyle should be original and accurate. Papers may be submitted in English or French, accompanied by a fifty-word abstract in the second language (even a rough translation will help the editors). All submissions will be evaluated by the Editor and anonymous readers.

Submit all papers in triplicate, typed double-spaced on one side only of 8 $x$-11-inch paper. Articles should not exceed 4000 words. Include all references on separate pages at the end of the article, following one of these standard formats: Chicago; MLA; APA. Graphics should be in cameraready form. Include a brief biographical sketch on a separate page with your submission.

Please include a self-addressed stamped envelope with your submission.

Submissions should be sent to:

Editor, Technostyle

Centre for Administrative and Information Studies

Room 8322

Faculty of Social Science

University of Western Ontario

London, Ontario

N6A 5 C2 


\section{GUIDE DE L'AUTEUR}

Technostyle publie, a l'intention des professeurs et des praticiens de la communication technique et professionnelle, des articles, de courtes notes et des commentaires. Les articles présentent le résultat de recherches et des analyses en profondeur; les notes, moins importantes, traitent d'une technique d'enseignement ou de rédaction déterminate. Les manuscrits dont le contenu doit être original et exact - peuvent être remis soit en anglais soit en français; il est demandé aux auteurs d'y joindre un résumé d'une cinquantaine de mots dans l'autre langue - ou encore une traduction qui, même approximative, sera utile à la Rédaction. Tous les manuscrits sont évalués par le Rédacteur en chef et des lecteurs anonymes.

Les manuscrits doivent être remis en triple exemplaire et dactylographiés à interligne double-et au recto seulement-sur papier format $8 \neq x 11$. Les articles ne doivent pas dépasser $\mathbf{4 0 0 0}$ mots. Les références doivent être regroupées a la fin de l'article sur des pages séparés et respecter l'un des protocoles bibliographiques suivants: Chicago, MLA, APA. Les graphiques doivent être prêts a photographier.

Les auteurs sont priés de joindre à leur manuscrit une courte notice biographique (sur une feuille séparée) et une enveloppe timbrée à leur adresse.

Les manuscrits soumis pour publication doivent être adressés à:

Editor, Technostyle

Centre for Administrative and Information Studies

Room 8322

Faculty of Social Science

University of Western Ontario

London, Ontario

N6A 5C2 